\title{
Características reprodutivas e suas associações com outras características de importância econômica na raça Nelore
}

[Reproductive traits and their associations with other economically important traits in Nelore]

\author{
M.J. Yokoo ${ }^{1}$, C.U. Magnabosco ${ }^{2}$, G.J.M. Rosa ${ }^{3}$, R.B. Lôbo ${ }^{4}$, L.G. Albuquerque ${ }^{5}$ \\ ${ }^{1}$ Empresa Brasileira de Pesquisa Agropecuária - Embrapa Pecuária Sul/ CPPSUL - Bagé, RS \\ ${ }^{2}$ Empresa Brasileira de Pesquisa Agropecuária - Embrapa Cerrados/CNPAF - Planaltina, DF \\ ${ }^{3}$ Department of Dairy Science - University of Wisconsin - Madison - WI, 53706, USA \\ ${ }^{4}$ Associação Nacional de Criadores e Pesquisadores - Ribeirão Preto, SP \\ ${ }^{5}$ Universidade Estadual Paulista - Faculdade de Ciências Agrárias e Veterinárias - Jaboticabal, SP
}

\section{RESUMO}

Objetivou-se com este trabalho estimar as herdabilidades $\left(h^{2}\right)$ e as correlações genéticas $\left(r_{g}\right)$ entre idade ao primeiro parto (IPP) e primeiro intervalo de partos (PIEP) e outras características como peso (PS) ao ano (A) e ao sobreano (S), altura do posterior (ALT) e perímetro escrotal (PE450) em animais da raça Nelore. Os parâmetros genéticos foram estimados em uma análise multicaracterística por modelo animal, utilizando-se a inferência bayesiana via algoritmo de "Gibbs Sampling". Os parâmetros genéticos estimados sugerem a existência de variabilidade genética para IPP $\left(h^{2}=0,26\right)$, sendo que a seleção para a diminuição da IPP de fêmeas Nelore deve responder à seleção individual, sem causar antagonismo do valor genético dos animais para PS $\left(r_{g}=-0,22\right.$ (A) e $\left.-0,44(\mathrm{~S})\right)$ e PE450 $\left(r_{g}=0,02\right)$. A seleção para a diminuição da IPP, no longo prazo, pode levar a um aumento da ALT dos animais, embora essa associação seja relativamente baixa $(-0,35)$. A estimativa de herdabilidade a posteriori para a característica PIEP foi baixa, $0,11 \pm 0,03$. As $\mathrm{r}_{\mathrm{g}}$ entre PIEP e as demais características estudadas indicam que a seleção para essas características de crescimento não afetará o PIEP

Palavras-chave: altura do posterior, componentes de variância, correlação genética, herdabilidade, precocidade sexual

\begin{abstract}
Heritability $\left(h^{2}\right)$ and genetic correlations $\left(r_{g}\right)$ were estimated between reproductive traits such as age at first calving ( $A F C)$, first calving interval ( $F C I)$ and other economically relevant traits, i.e., weight $(W)$ at year $(Y)$ and at 18 months of age (S), scrotal circumference (SC), and hip height $(H H)$ in Nelore cattle. The genetic parameters were estimated in a multiple-trait analysis, with animal models using the Bayesian inference by Gibbs Sampling algorithm. The genetic parameters estimated in this work suggest the existence of genetic variability for AFC $\left(h^{2}=0.26\right)$, where the selection for the reduction of Nelore females AFC should respond to mass selection, without causing genetic antagonism in the selection of $W$ $\left(r_{g}=-0,22(Y)\right.$ and $\left.-0,44(S)\right)$, and $S C\left(r_{g}=0,02\right)$. The selection for the AFC in the long term could lead to an increase in the animal's frame, although this association is relatively low (-0.35). The posteriori heritability estimate for $F C I$ was low, $0.11 \pm 0.03$. The $r_{g}$ between $F C I$ and the other traits studied indicate that selection for these growth traits will not affect the FCI.
\end{abstract}

Keywords: genetic correlation, heritability, hip height, variance components, sexual precocity

Recebido em 1 de março de 2011

Aceito em 28 de setembro de 2011

E-mail: yokoomarcos@hotmail.com

Apoio financeiro:CNPq-Brasil, CAPES, FAPESP e ANCP 


\section{INTRODUÇÃO}

Em áreas tropicais, durante as últimas décadas, diversos programas de melhoramento genético que desenvolvem tecnologias e oferecem serviços de avaliação genética em gado de corte têm sido implantados. Grande ênfase é dada no desenvolvimento e na aplicação de métodos estatísticos adequados para avaliação genética de animais sob seleção, o que tem elevado os índices de produtividade da pecuária de corte. Entretanto, em vários destes programas, são priorizadas as características peso vivo do animal (PS) e o perímetro escrotal (PE), sem avaliar as suas associações com outras medidas importantes em sistemas de produção extensivos.

Rebanhos detentores de elevada precocidade sexual e fertilidade possuem maior disponibilidade de animais, tanto para venda, como para seleção, permitindo maior intensidade seletiva e, consequentemente, progressos genéticos mais elevados e maior lucratividade (Toelle e Robison, 1985). Contudo, em poucos programas de melhoramento genético de bovinos de corte têm-se utilizado características reprodutivas mensuradas em fêmeas como critério de seleção, sendo o PE uma das poucas características indicadoras de reprodução empregadas.

A redução da idade ao primeiro parto (IPP) pode afetar significativamente a produtividade do rebanho, pela sua influência na produção de bezerros durante a vida útil da matriz, possibilitando uma intensidade maior de seleção e reduzindo o intervalo de gerações, com consequente aumento do progresso genético e da lucratividade para todo o sistema de produção. Outra característica reprodutiva interessante para melhorar a fertilidade do rebanho é o primeiro intervalo de partos (PIEP), pois este leva em conta a habilidade da fêmea de reconceber o mais rápido possível após o primeiro parto, o que, hoje, é ainda um ponto de estrangulamento na produção de gado de corte em áreas tropicais, em sistemas de produção extensivos.

Geralmente, características reprodutivas mensuradas em fêmeas da raça Nelore apresentam baixas estimativas de herdabilidade, em torno de 0,10 (Dias et al., 2004ab; Boligon et al., 2007; 2008), sendo fortemente influenciadas por fatores ambientais. No entanto, estimativas de herdabilidade de magnitudes moderadas, variando entre 0,28 e 0,37 para IPP (Mercadante et al., 2000; Bertazzo et al., 2004) e de 0,32 a 0,42 para IEP (Campello et al., 1999; Silveira et al., 2004) são relatadas. Considerando que essas características são fundamentais para a manutenção e seleção do rebanho, e que os ganhos obtidos pelo melhoramento genético das mesmas serão permanentes, pode-se justificar o investimento na seleção de animais geneticamente superiores. Assim, estudos da associação genética das características reprodutivas obtidas em fêmeas com outras de importância econômica em áreas tropicais podem trazer informações para melhor planejar os critérios e os objetivos de seleção, de acordo com cada sistema e o mercado alvo. Além disso, pode-se melhor entender os fatores que afetam o desempenho sexual de fêmeas jovens da raça Nelore. Dessa maneira, o objetivo deste trabalho foi estimar as herdabilidades e as correlações genéticas entre as características reprodutivas, idade ao primeiro parto e primeiro intervalo de partos, e outras características de importância econômica, como peso (PS) ao ano (A) e ao sobreano (S), altura do posterior ao sobreano (ALT) e perímetro escrotal ao sobreano (PE450), em animais da raça Nelore.

\section{MATERIAL E MÉTODOS}

O presente trabalho foi desenvolvido com animais da raça Nelore pertencentes aos rebanhos de criadores associados ao Programa de Melhoramento Genético da Raça Nelore - Nelore Brasil (PMGRN) da Associação Nacional de Criadores e Pesquisadores de Ribeirão Preto, SP (ANCP), conjuntamente com a Universidade Estadual Paulista (UNESP), Campus de Jaboticabal, SP. Foram avaliadas as características reprodutivas: idade ao primeiro parto (IPP), obtida pela diferença entre a data do primeiro parto e a data do nascimento da fêmea, em dias, e primeiro intervalo de partos (PIEP), medida pela diferença entre o primeiro e o segundo parto, em dias. Essas características reprodutivas mensuradas em fêmeas são provenientes de quatro rebanhos do PMGRN de matrizes nascidas entre 1977 e 2003 . Verificouse ainda, a existência de nascimento dos bezerros ao longo de todo o ano, embora uma parte das fazendas adotasse um longo período de estação de monta na época das águas. 
Durante quatro anos, foram tomados 2.966 registros de pesos ao ano e ao sobreano (PS_A e PS_S) e 2.356 medidas de altura do posterior ao sobreano (ALT) dos animais desses rebanhos. Além disso, 5.695 medidas de perímetro escrotal ao sobreano, padronizadas aos 450 dias de idade (PE450), foram analisadas de animais nascidos entre os anos de 1998 e 2003.

O grupo de contemporâneos (GC) foi definido como animais da mesma fazenda, sexo (exceto para PE450, IPP e PIEP), estação e ano de nascimento e lote de manejo. Além disso, para IPP e PIEP, acrescentaram-se as variáveis fazenda e estação do parto na formação do GC. Foram eliminados os GC, que agregavam apenas animais filhos de um mesmo touro, além de GC, com menos de três animais. Na Tab. 1, pode-se observar a estrutura do arquivo dos dados, após consistência.

Tabela 1. Descrição do arquivo dos dados, de bovinos da raça Nelore

\begin{tabular}{lccccc}
\hline Características $^{(1)}$ & $\mathrm{N}^{\mathrm{o}}$ & Média (DP) & $\mathrm{N}^{\mathrm{o}}$ Touros & $\mathrm{N}^{\mathrm{o}}$ Mães & $\mathrm{N}^{\mathrm{o}} \mathrm{GC}$ \\
\hline PS_A, kg & 2.358 & $268,97(64,09)$ & 227 & 2.079 & 204 \\
PS_S, kg & 2.966 & $339,69(65,98)$ & 236 & 2.683 & 302 \\
ALT, cm & 2.356 & $136,06(5,04)$ & 226 & 2.308 & 250 \\
PE450, mm & 5.695 & $245,87(30,22)$ & 386 & 4.109 & 188 \\
IPP, dias & 7.648 & $1.117(161,98)$ & 485 & 6.785 & 706 \\
PIEP, dias & 5.764 & $466(123,80)$ & 439 & 4.437 & 656 \\
\hline
\end{tabular}

(1) PS_A e PS_S = peso vivo obtido ao ano e ao sobreano, respectivamente; ALT = altura do posterior mensurada ao sobreano; PE450 = perímetro escrotal ao sobreano padronizado aos 450 dias de idade; IPP = idade ao primeiro parto; $\mathrm{PIEP}=$ primeiro intervalo entre partos; $\mathrm{N}^{\mathrm{o}}=$ número de animais; $\mathrm{DP}=$ desvio-padrão; $\mathrm{N}^{\mathrm{o}}$ Touros = número de touros (pais); $\mathrm{N}^{o}$ Mães = número de vacas (mães); $\mathrm{N}^{\circ} \mathrm{GC}=$ número de grupos de contemporâneos;

Os componentes de (co)variância foram estimados em uma análise multicaracterística por meio do modelo animal, utilizando-se o programa GIBBS2F90, desenvolvido por Misztal et al. (2002), que foi escrito em linguagem FORTRAN 90 e utiliza a inferência bayesiana pelo algoritmo da amostragem de Gibbs. Para os valores a priori das (co)variâncias aditivas e residuais, foi utilizada a distribuição não informativa ou "flat" (não refletindo conhecimento prévio do parâmetro), que o programa transforma em uma distribuição imprópria. Foi assumida uma distribuição uniforme a priori para os efeitos sistemáticos, e para os demais componentes, a distribuição Wishart invertida.

Inicialmente, foi computada uma cadeia de 1.500.000 iterações, sendo que o tamanho final da cadeia bem como a especificação do "burn-in" e do intervalo de amostragem ("thin") foram definidos por meio do critério de Raftery e Lewis (1992), o qual indica a convergência da cadeia pela baixa correlação serial existente entre os ciclos, e também pelo critério de Gelman e Rubin (1992). Essas análises foram feitas utilizando-se o pacote "BOA" ("Bayesian Output Analysis") do programa R (R a language...,
2006). Além desses critérios, a convergência da cadeia de Gibbs também foi verificada por meio de inspeção visual dos parâmetros pelo gráfico de dispersão. Depois de verificada a convergência da cadeia de Gibbs, as estimativas da distribuição a posteriori foram calculadas após um descarte de 600.000 iterações iniciais e uma amostragem a cada doze iterações, totalizando 75.000 amostras para a estimativa da média, desvio-padrão, mediana, moda, erro de Monte Carlo, tamanho efetivo da amostra e intervalo de $95 \%$ da maior densidade a posteriori (HPD). Foram utilizados os pacotes "BOA" e "CODA" ("Output Analysis and Diagnostics for MCMC", Plummer et al., 2006) do programa R para esse fim.

A matriz de parentesco foi até a última geração conhecida e totalizou 69.872 animais. Os modelos para todas as características incluíram os efeitos aleatórios genético aditivo direto e residual, os efeitos sistemáticos do GC e da idade do animal como covariável (efeitos linear e quadrático, exceto para o PE450 e IPP). A característica PIEP foi analisada pelo mesmo modelo anterior, apenas acrescentando o efeito sistemático do tipo de cobrição para a segunda prenhez (inseminação ou monta natural). Para as 
características PS_A, PS S e ALT, também acrescentou-se o efeito sistemático da idade da vaca ao parto, em seis classes $(35 ; 36$ a $47 ; 48$ a 59; 60 a 71; 72 a 119 e > 120 meses). O modelo geral utilizado pode ser representado em notação matricial como: $\mathrm{y}=\mathbf{X} \beta+\underset{\sim}{\mathbf{Z}} \mathbf{a}+\underset{\sim}{\mathrm{e}}$, em que $\mathrm{y}$ é

o vetor de observações; $\beta$ é o vetor de efeitos sistemáticos desconhecidos; a é o vetor de efeitos aleatórios desconhecidos que representam os valores genéticos aditivos de cada animal; e é o vetor de efeitos aleatórios residuais desconhecidos; e $\mathbf{X}$ e $\mathbf{Z}$ são as matrizes de incidência, que relacionam os registros aos efeitos fixos e aos aleatórios genéticos aditivos, respectivamente. As pressuposições acerca da distribuição de $\mathrm{y}$, a e e podem ser descritas como:

$$
\mathrm{y} \sim \mathrm{N}\left(\mathbf{X} \beta+\underset{\sim}{\mathbf{Z}}, \mathbf{I} \sigma_{\mathrm{e}}^{2}\right), \quad \mathrm{p}\left(\underset{\sim}{\mathrm{a}} \mid \sigma_{\mathrm{a}}^{2}\right) \sim \mathrm{N}(0, \mathbf{A} \otimes \mathbf{G}), \mathrm{e}
$$

$\mathrm{p}\left(\mathrm{e} \mid \sigma_{\mathrm{e}}^{2}\right) \sim \mathrm{N}\left(0, \mathbf{I} \sigma_{\mathrm{e}}^{2}\right)$, em que $\mathbf{A}$ é a matriz de

numeradores dos coeficientes de parentesco de Wright entre os animais; I é a matriz identidade de ordem igual à dimensão linha de $\mathrm{y} ; \mathbf{G}$ é a matriz de (co)variância genética aditiva e $\sigma_{\mathrm{a}}^{2}$ e $\sigma_{\mathrm{e}}^{2}$ são as variâncias aditivas e residuais, respectivamente.

\section{RESULTADOS E DISCUSSÃO}

Os valores a posteriori das medidas descritivas de cada parâmetro estimado, como a média, a mediana e a moda foram similares. Os erros de Monte Carlo foram baixos, e os tamanhos efetivos de amostras altos, maiores que 300 (estatísticas não apresentadas) de todos os parâmetros estudados. Essas estatísticas descritivas indicam confiabilidade nos parâmetros estimados e convergência da análise.

Assumindo que estimativas de herdabilidade baixa vão até $20 \%$, e moderada, de 20 a $40 \%$ (Bourdon, 1997), pode-se observar, na Fig. 1, que a maioria dos valores estimados para PIEP e IPP encontram-se nas magnitudes baixa e moderada, respectivamente. A herdabilidade média a posteriori da característica IPP foi de magnitude moderada, $0,26 \pm 0,05$, e a HPD variou de 0,18 a 0,34 . Em sistemas de produção em que animais sexualmente precoces são mais eficazes, como, por exemplo, propriedades com escassez de alimento e água e produtores que desejam animais de ciclo de produção mais curto, a seleção de animais como pais de futuras gerações com valores genéticos negativos para IPP e PIEP, por exemplo, poderá ser utilizada como ferramenta em programas de melhoramento animal, visto que a IPP é uma característica que deverá responder à seleção massal, em rebanhos da raça Nelore. Este resultado corrobora com estudos em animais da raça Nelore (Mercadante et al., 2000; Dias et al., 2004a e b; Azevedo et al., 2006).

No presente estudo, vale ressaltar que somente algumas novilhas tiveram sua primeira oportunidade de emprenhar ao redor dos 16 meses de idade, e aquelas que não conceberam tiveram uma segunda chance em torno dos dois anos de idade, o que ocorreu com a maioria das fêmeas (95\%). Silva e Albuquerque (2004) verificaram que a seleção para precocidade sexual de fêmeas Nelore deveria ser feita em idades mais jovens, pois, quando as fêmeas são expostas antecipadamente à reprodução, existem maiores possibilidades de um rápido ganho genético, pois a herdabilidade da característica prenhez aos 18 meses de idade foi maior $(0,52 \pm 0,08)$ que para prenhez aos 27 meses $(0,12 \pm 0,02)$. Além disso, é importante verificar a definição do grupo de contemporâneos, como foi discutido por Dias et al. (2004a e b). Segundo esses autores, a inclusão de todas as fêmeas nascidas no rebanho, e não só as que foram colocadas em reprodução, proporciona aumento da variabilidade genética para IPP. Dias et al. (2004b) também concluíram que a inclusão de ano e estação do parto na formação de grupos contemporâneos provavelmente diminuiu parte da variação genética existente para IPP. Outro fato importante que se deve levar em conta ao se desafiar as novilhas é a definição da época mais apropriada da entrada em reprodução dessas fêmeas, pois envolve uma série de particularidades complexas. Todos esses fatores, juntamente com o modelo utilizado para estimação do valor genético, são os que podem definir a velocidade do ganho genético para IPP. 


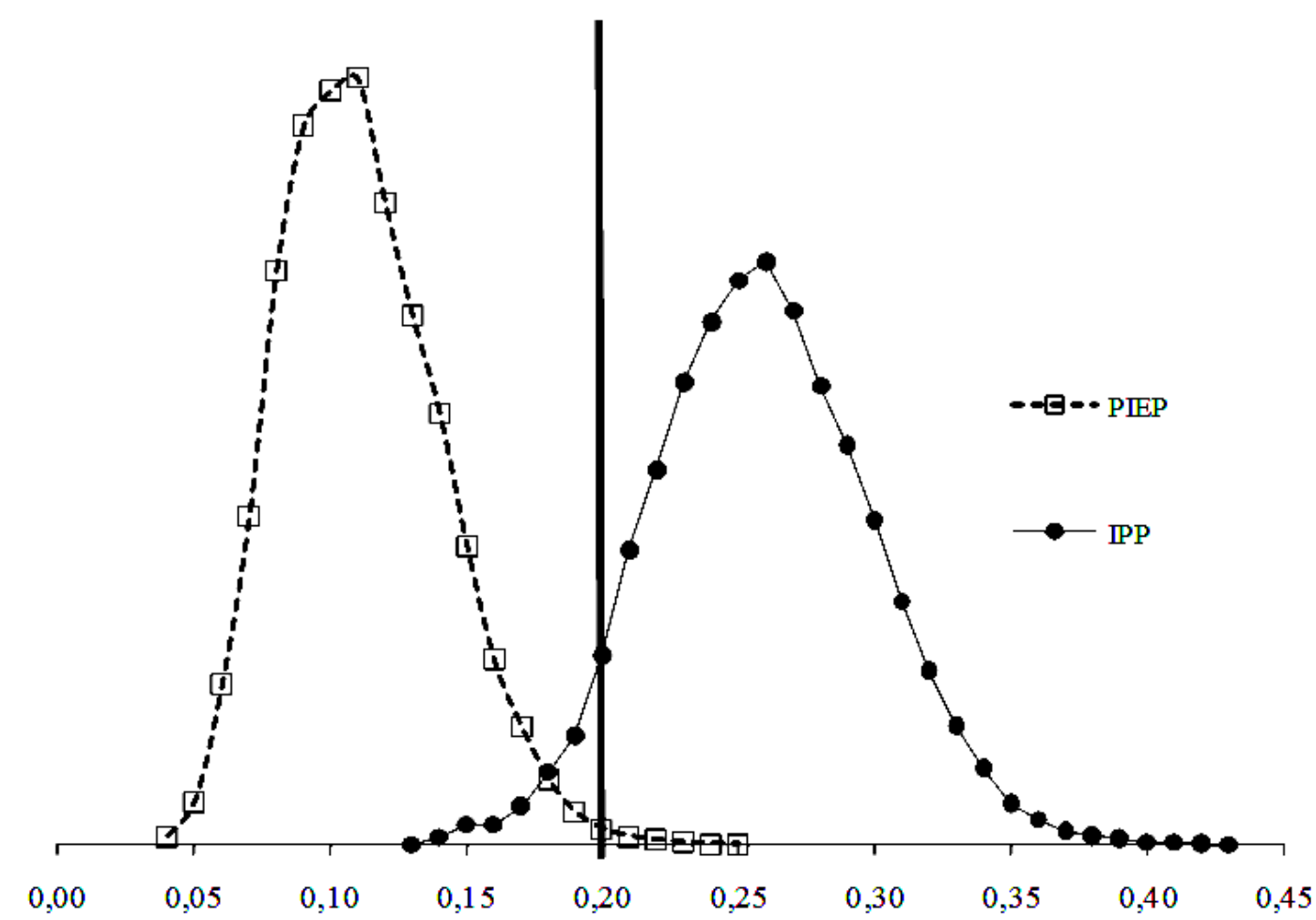

Figura 1. Densidade marginal a posteriori das estimativas de herdabilidades das características idade ao primeiro parto (IPP) e primeiro intervalo entre partos (PIEP) da raça Nelore, juntamente com o critério de magnitudes baixas e moderadas sugerido por Bourdon (1997).

A estimativa de herdabilidade média a posteriori (Fig. 1) da característica PIEP foi de magnitude baixa, $0,11 \pm 0,03$, e a HPD de 0,06 a 0,17 , sugerindo que a utilização dessa característica como critério de seleção deverá promover lento progresso genético para eficiência reprodutiva em rebanhos da raça Nelore. Este resultado é similar aos relatados em animais da raça Nelore (Gressler et al., 2000; Mercadante et al., 2000). Por outro lado, Campello et al. (1999) e Silveira et al. (2004), ao estudarem a característica intervalo de partos, considerando várias medidas por animal, estimaram coeficientes de herdabilidade de magnitudes moderadas a altas, 0,32 e 0,42 , respectivamente. De acordo com esses autores, quando se avalia somente o primeiro intervalo de partos, ou os primeiros, obtêm-se coeficientes de herdabilidade de baixa magnitude, principalmente devido à maior vulnerabilidade e ao estresse nutricional a que as vacas jovens são submetidas, fazendo com que as diferenças genéticas sejam de difícil identificação. Alguns autores, dentre eles Bourdon e Brinks (1983), Azzam et al. (1986) e
Marshall et al. (1990) discutem as limitações da característica intervalos de partos, pois a existência da estação de monta torna os dados que são coletados neste período truncados, sendo estes tratados de forma incorreta pelos modelos lineares que normalmente são utilizados. Outro problema é que a estação de monta pode acabar, e o animal, por algum outro fator ambiental, pode não ter emprenhado, fato que provavelmente irá atrapalhar o desempenho da novilha que supostamente teria condições de conceber. Dessa forma, a baixa estimativa de variabilidade genética do PIEP, encontrada no presente trabalho, sugere o emprego dos modelos de sobrevivência (Ducrocq e Solkner, 1998) para a avaliação desse caractere, os quais permitem incluir, na análise, dados de animais que entraram na estação de monta e que não tiveram o registro de parto anotado.

As associações entre IPP e PIEP não foram diferentes da nulidade, sendo que as correlações genéticas e fenotípicas foram de $-0,12 \pm 0,17$ e $0,02 \pm 0,03$, respectivamente, indicativo de que a seleção para diminuição da IPP não deverá levar 
à diminuição do PIEP, por resposta correlacionada. Esta correlação genética é similar à relatada por Mercadante et al. (2000), em animais da raça Nelore $(-0,06)$.

Na Tab. 2, estão apresentadas as médias, os desvios-padrão e as HPD das estimativas $a$ posteriori das correlações genéticas e fenotípicas entre as características reprodutivas, IPP e PIEP e as demais características analisadas. Em geral, as correlações entre PIEP e as demais características estudadas não foram diferentes de zero. Em áreas tropicais, poucos estudos relativos à associação do PIEP e caracteres de crescimento para raças zebuínas são encontrados. Martins Filho e Lôbo (1994) relataram valores similares $(-0,04 \pm 0,25)$ aos deste estudo para correlação genética entre intervalo de partos e PE em animais da raça Nelore. Esses resultados indicam que a seleção para diminuir PIEP não afetará o crescimento dos animais e vice-versa, por resposta correlacionada.

Tabela 2. Estimativas a posteriori das correlações genéticas $\left(\hat{r}_{g}\right)$ e fenotípica $\left(\hat{r}_{f}\right)$ entre as características reprodutivas, idade ao primeiro parto (IPP) e primeiro intervalo entre partos (PIEP) e peso ao ano (PS_A), peso ao sobreano (PS_S), altura do posterior ao sobreano (ALT) e perímetro escrotal padronizado aos 450 dias de idade (PE450), em animais da raça Nelore

\begin{tabular}{|c|c|c|c|c|c|}
\hline Características & & PS_A, kg & PS_S, kg & $\mathrm{ALT}, \mathrm{cm}$ & $\mathrm{PE} 450, \mathrm{~mm}$ \\
\hline \multirow{4}{*}{ IPP, dias } & & $-0,22 \pm 0,16^{(1)}$ & $-0,44 \pm 0,17$ & $-0,35 \pm 0,11$ & $0,04 \pm 0,08$ \\
\hline & $r_{g}$ & $-0,52$ a $0,10^{(2)}$ & $-0,79$ a $-0,13$ & $-0,55$ a $-0,14$ & $-0,13$ a 0,21 \\
\hline & $\hat{r}$ & $0,09 \pm 0,06$ & $0,02 \pm 0,06$ & $0,09 \pm 0,05$ & $0,02 \pm 0,03$ \\
\hline & $r_{f}$ & $-0,03$ a 0,20 & $-0,10$ a 0,13 & 0,04 a 0,19 & $-0,04$ a 0,07 \\
\hline \multirow{4}{*}{ PIEP, dias } & $r$ & $0,06 \pm 0,16$ & $0,04 \pm 0,16$ & $-0,02 \pm 0,22$ & $-0,02 \pm 0,13$ \\
\hline & $r_{g}$ & $-0,26$ a 0,34 & $-0,24$ a 0,37 & $-0,41$ a 0,40 & $-0,28$ a 0,23 \\
\hline & $\hat{r}$ & $-0,43 \pm 0,08$ & $-0,26 \pm 0,06$ & $-0,05 \pm 0,32$ & $0,00 \pm 0,03$ \\
\hline & $r_{f}$ & $-0,56$ a $-0,28$ & $-0,35$ a $-0,13$ & $-0,53$ a 0,56 & $-0,06$ a 0,05 \\
\hline
\end{tabular}

${ }^{(1)}$ Estimativas a posteriori da média \pm erro-padrão; ${ }^{(2)}$ Estimativas do intervalo de $95 \%$ de maior densidade a posteriori.

Atualmente, o PE também é considerado como uma característica indicadora de precocidade sexual em programas de melhoramento genético, sendo ainda relacionado de forma desejável com diversas características de machos e fêmeas (Silva et al., 2000; Pereira et al., 2000 e 2002) e, dessa forma, vem sendo bastante utilizado como critério de seleção. Portanto, poder-se-ia esperar que animais com melhores valores genéticos para PE450 também o fossem para IPP e PIEP, pois o PE possui correlações genéticas favoráveis com idade à puberdade em machos e fêmeas (Martin et al., 1992; Moser et al., 1996). No entanto, no presente estudo, as estimativas de correlações genéticas entre o PE450 e as duas características reprodutivas mensuradas em fêmeas, IPP e PIEP, foram baixas (Tab. 2), mas não diferentes da nulidade $\quad(0,04 \pm 0,08$ e $-0,02 \pm 0,13$, respectivamente). Este achado discorda de vários resultados descritos na literatura, como os de Martins Filho e Lôbo (1991), Gressler et al. (2000) e Pereira et al. (2000 e 2001), que estimaram valores negativos para essas correlações genéticas, variando de $-1,00$ a $-0,22$, em bovinos Nelore. Contudo, Gressler et al.
(2000), Pereira et al. (2000 e 2001) e Pereira $e t$ al. (2010) reportaram herdabilidades próximas de zero para IPP e PIEP, variando de 0,01 a 0,12 ; além disso, Martins Filho e Lôbo (1991) utilizaram o modelo touro e o método dos quadrados mínimos para estimação dos componentes de (co)variância, diferentemente do presente trabalho.

Estudando a correlação genética entre probabilidade de prenhez de novilhas e PE em animais Nelore criados em regiões tropicais, Eler et al. (2004) descreveram uma associação favorável entre elas, embora de baixa magnitude, 0,20. Toelle e Robinson (1985) relataram correlações genéticas entre PE e IPP variando de $-0,14$ a $-0,38$ para animais Hereford, sendo que essa correlação variou conforme foi definida a característica IPP, pois, quando os autores consideravam somente as novilhas que pariram em torno dos dois anos, essa correlação era baixa $(-0,14)$ e, quando consideravam todas as novilhas que pariram em torno de dois e três anos, esse parâmetro era de uma magnitude maior $(-0,38)$. 
Na Fig. 2, pode-se observar que a maioria das densidades das correlações genéticas entre IPP e as características ALT, PS_A e PS_S estão abaixo do zero, indicativo de que essas correlações são realmente negativas, embora a associação entre IPP e PS_A seja de baixa magnitude. As médias das estimativas $a$ posteriori das correlações genéticas entre os pesos (PS_A e PS_S) e a IPP foram negativas e de magnitudes moderadas a baixas, sendo $-0,22$ e -0,44, respectivamente. Em animais da raça Nelore, Mercadante et al. (2000) e Shiotsuki et al. (2009) também relataram correlações genéticas favoráveis, embora de baixas magnitudes, entre características de precocidade sexual (IPP e probabilidade de prenhez precoce, nessa ordem) e PS_A, -0,23 e 0,08, respectivamente. Essas estimativas indicam que a seleção para o aumento do peso, em longo prazo, deve levar a uma diminuição da IPP, por resposta correlacionada, sendo que essa resposta deverá ser mais efetiva quando a seleção for praticada mais intensivamente para o PS_S.

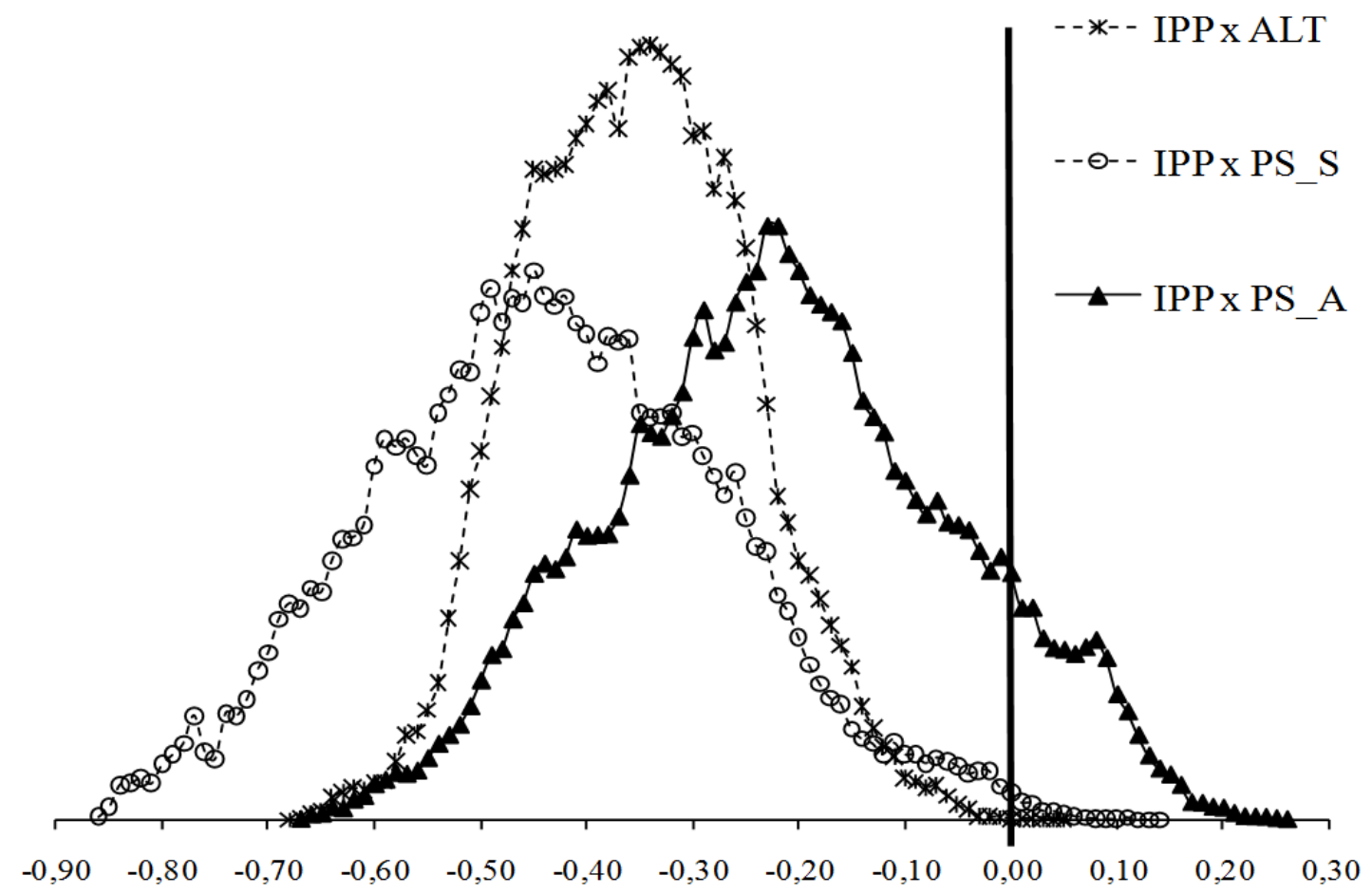

Figura 2. Densidade marginal a posteriori das estimativas de correlações genéticas entre as características idade ao primeiro parto (IPP) e altura do posterior (ALT) e peso ao ano e sobreano (PS_A e PS_S) da raça Nelore.

A estimativa da média a posteriori da correlação genética entre ALT e IPP foi negativa e de magnitude baixa $(-0,35)$. Esse resultado sugere que a seleção para a diminuição da IPP, em longo prazo, poderia aumentar a estatura dos animais, por resposta correlacionada. Resultados semelhantes, embora de menor magnitude, foram estimados por Silva et al. (2003), para a correlação genética entre ALT e probabilidade de prenhez de novilhas aos 14 meses de idade $(0,10)$, também em animais Nelore criados em áreas tropicais. Por outro lado, Vargas et al.
(1998), analisando a relação entre a ALT e a idade à puberdade em fêmeas Brahman, estimaram correlação baixa e positiva $(0,25)$, ao contrário do presente estudo, concluindo que um aumento do tamanho dos animais poderá levar ao atraso do início da vida reprodutiva das fêmeas, no sistema de produção em áreas temperadas. A correlação genética negativa entre IPP e ALT no presente trabalho (Fig. 2 e Tab. 2) provavelmente se deve bastante à associação genética entre ALT e PS_S, que é positiva e de magnitude moderada (0,59, resultado não apresentado). Assim sendo, 
a seleção para aumento do PS_S e a diminuição da IPP deverão, por resposta correlacionada, levar a um aumento da estatura do animais em animais da raça Nelore criados em sistemas tropicais. Portanto, um índice de seleção envolvendo essas três características deveria ser estudado.

Com exceção da estimativa a posteriori das correlações fenotípicas entre IPP e PS (A e S), todas as outras estimativas de correlações fenotípicas apresentaram o mesmo comportamento que as correlações genéticas. As correlações fenotípicas entre IPP e PS (A e S) foram positivas, porém muito próximas de zero $(0,09$ e 0,02 , respectivamente). Essa inversão de direção das correlações genéticas com as fenotípicas, entre IPP e PS (A e S), pode ser explicada pela negativa e moderada correlação residual entre essas características (em torno de 0,40). As estimativas de correlações fenotípicas entre PIEP e as características de crescimento não foram diferentes de zero, com exceção da correlação entre PIEP e PS (A e S), sugerindo que animais mais pesados terão um menor PIEP.

\section{CONCLUSÕES}

A seleção para a diminuição da idade ao primeiro parto de fêmeas Nelore deverá responder à seleção massal e poderá aumentar o peso corporal ao ano e ao sobreano dos animais, por resposta correlacionada. A seleção para o aumento do perímetro escrotal aos 450 dias de idade não afetará a idade ao primeiro parto e nem o primeiro intervalo de partos de fêmeas Nelore, por resposta correlacionada. A seleção praticada para as características de crescimento, em longo prazo, não deverá afetar o primeiro intervalo de partos, por resposta correlacionada.

\section{AGRADECIMENTOS}

Ao Conselho Nacional de Desenvolvimento Científico e Tecnológico e à Fundação de Amparo à Pesquisa do Estado de São Paulo, pelo apoio financeiro. À Associação Nacional de Criadores e Pesquisadores de Ribeirão Preto, SP, pela parceria na realização deste trabalho. Às fazendas que colaboraram para realização deste trabalho: Bacuri, Guaporé Pecuária do Grupo OB, Grupo Hora, Rancho da Matinha, Colonial, São Dimas, Remanso, Passa Quatro e Santa Marta.

\section{REFERÊNCIAS}

AZEVEDO, D.M.M.R.; MARTINS FILHO, R.; LOBO R.N.B. et al. Desempenho reprodutivo de vacas Nelore no Norte e Nordeste do Brasil. Rev. Bras. Zootec., v.35, supl., p.988-996, 2006.

AZZAM, S.M.; PENNEL, P.L.; KINDER, J.E. et al. Estimation of expected time to puberty in terminated experiments using survival analysis. J. Anim. Sci., v.63, supl. 1, p.129. 1986.

BERTAZZO, R.P.; FREITAS, R.T.F.; GONÇALVES, T.M. et al. Parâmetros genéticos de longevidade e produtividade de fêmeas da raça Nelore. Rev. Bras. Zootec., v.33,p. 11181127, 2004.

BOLIGON, A.A.; ALBUQUERQUE, L.G.; RORATO, P.R.N. Associações genéticas entre pesos e características reprodutivas em rebanhos da raça Nelore. Rev. Bras. Zootec., v.37, p.596$601,2008$.

BOLIGON, A.A.; RORATO, P.R.N.; ALBUQUERQUE, L.G. Correlações genéticas entre medidas de perímetro escrotal e características produtivas e reprodutivas de fêmeas da raça Nelore. Rev. Bras. Zootec., v.36, p.565-571, 2007.

BOURDON, R.M. Understanding animal breeding. New Jersey: Colorado State University, 523p., 1997.

BOURDON, R.M.; BRINKS, J.S. Calving date versus calving interval as a reproductive measure in beef cattle. J. Anim. Sci., v.57, p.14121417,1983 .

CAMPELLO, C.C.; MARTINS FILHO, R.; LOBO, R.N.B. Intervalos de parto e fertilidade real em vacas Nelore no estado do Maranhão. Rev. Bras. Zootec., v.28, p.474-479, 1999.

DIAS, L.T.; EL FARO, L.; ALBUQUERQUE, L.G. Efeito da idade de exposição de novilhas à reprodução sobre estimativas de herdabilidade da idade ao primeiro parto em bovinos Nelore. Arq. Bras. Med. Vet. Zootec., v.56, p.370-373, 2004a.

DIAS, L.T.; EL FARO, L.; ALBUQUERQUE, L.G. Estimativas de herdabilidade para idade ao primeiro parto de novilhas da raça Nelore. Rev. Bras. Zootec., v.33, p.97-102, 2004 b. 
DUCROCQ, V.; SOLKNER, J. The survival kit (v. 3.0): a Fortran package for the analysis of survival data. In: WORLD CONGRESS OF GENETIC APPLIED TO LIVESTOCK PRODUCTION, 6., Armidale Austrália, 1998. Proceedings ... Armidale: [s.n.], 1998.

ELER J.P.; SILVA J.A.IIV.; EVANS J.L. et al. Additive genetic relationship between heifer pregnancy and scrotal circumference in Nellore cattle. J. Anim. Sci., v.82, p.2519-2527, 2004.

GELMAN, A.; RUBIN, D.B. Inference from iterative simulation using multiple sequence. Stat. Sci., v.7, p.457-511, 1992.

GRESSLER, L.S.; BERGMANN, J.A.G.; PEREIRA, C.S. et al. Estudo das associações genéticas entre perímetro escrotal e características reprodutivas de fêmeas Nelore. Rev. Bras. Zootec., v.29, p.427-437, 2000.

MARSHALL, D.M.; MINQIANG, W.; FREKING, B.A. Relative calving date of firstcalf heifers as related to production efficiency and subsequent reproductive performance. $J$. Anim. Scie., v.68, p.1812-1817, 1990.

MARTIN, L.C.; BRINKS, J.S.; BOURDON, R.M.; CUNDIFF, L.V. Genetic effects on beef heifer puberty and subsequent reproduction. $J$. Anim. Scie., v.70, p.4006-4017, 1992.

MARTINS FILHO, R.; LÔBO, R.B. Correlação genética entre circunferência escrotal e idade ao primeiro parto e intervalo entre partos. Rev. Cien. Agron., v.25, p.10-15, 1994.

MARTINS FILHO, R.; LÔBO, R.B. Estimates of genetic correlations between sire scrotal circumference and offspring age at first calving in Nellore cattle. Rev. Bras. Genet., v.14, p.209$212,1991$.

MERCADANTE, M.E.Z.; LÔBO, R.B.; OLIVEIRA, H.N. Estimação de (co) variâncias entre características de reprodução e de crescimento em fêmeas de um rebanho Nelore. Rev. Bras. Zootec., v.29, p.997-1004, 2000.

MISZTAL, I.; TSURUTA, S.; STRABEL, T. et al. BLUPF90 and related programs (BGF90). In: WORLD CONGRESS OF GENETIC APPLIED TO LIVESTOCK PRODUCTION, 7., Montpellier, France, 2002. Proceedings... Montpellier: Francis Minvielle, v.28, p.7, 2002.
MOSER, D.W.; BERTRAND, J.K.; BENYSHEK, L.L. et al. Effects of selection for scrotal circumference in Limousin bulls on reproductive and growth traits of progeny. $J$. Anim. Scie., v.74, p.2052-2057, 1996.

PEREIRA, E.; ELER, J.P.; COSTA, F.A.A.; FERRAZ, J.B.S. Análise genética da idade ao primeiro parto e do perímetro escrotal em bovinos da raça Nelore. Arq. Bras. Med. Vet. Zootec., v.53, p.116-121, 2001.

PEREIRA, E.; ELER, J.P.; FERRAZ, J.B.S. Análise genética de características reprodutivas na raça Nelore. Pesqui. Agropecu. Bras., v.37, p.703-708, 2002.

PEREIRA, E.; ELER, J.P.; FERRAZ, J.B.S. Correlação genética entre perímetro escrotal e algumas características reprodutivas na raça Nelore. Rev. Bras. Zootec., v.29, p.1676-1683, 2000.

PEREIRA, M.C.; YOKOO, M.J.; BIGNARDI, A.B. et al. Altura da garupa e sua associação com características reprodutivas e de crescimento na raça Nelore. Pesqui. Agropecu. Bras., v.45, p.613-620, 2010.

PLUMMER, M.; BEST, N.; COWLES, K.; VINES, K. 2006. Coda: output analysis and diagnostics for MCMC. R-project. [2006]. Disponível em:

<http://rh-mirror.linux.iastate.edu/CRAN/> Acessado em: 30 out. 2006.

$R$ : a language and environment for statistical computing. Vienna, Austria: R. Foundation for Statistical Computing, 2006. Disponível em: <http://www.R-project.org, 2006> Acessado em: 06 out. 2006.

RAFTERY, A.E.; LEWIS, S.M. One long run with diagnostics: implementation strategies for Markov chain Monte Carlo. Stat. Sci., v.7, p.493 - 497, 1992.

SHIOTSUKI, L.; SILVA, J.A.IIV.; TONHATI, H.; ALBUQUERQUE, L.G. Genetic associations of sexual precocity with growth traits and visual scores of conformation, finishing, and muscling in Nelore cattle. J. Anim. Sci., v.87, p.1591-1597, 2009. 
SILVA, A.M.; ALENCAR, M.M.; FREITAS, A.R. et al. Herdabilidades e correlações genéticas para peso e perímetro escrotal de machos e características reprodutivas e de crescimento de fêmeas, na raça Canchim. Rev. Bras. Zootec., v.29, supl. 2, p.2223-2230, 2000.

SILVA, J.A.IIV.; ALBUQUERQUE, L.G. Estudo genético da precocidade sexual de novilhas em um rebanho Nelore. In: REUNIÃO ANUAL DA SOCIEDADE BRASILEIRA DE ZOOTECNIA, 41., 2004, Campo Grande, MS. Anais... Campo Grande, MS: SBZ, 2004. (CDROM).

SILVA, J.A.IIV.; VAN MELIS, M.H.; ELER, J.P.; FERRAZ, J.B.S. Estimação de parâmetros genéticos para probabilidade de prenhez aos 14 meses e altura na garupa em bovinos da raça Nelore. Rev. Bras. Zootec., v.32, p.1141-1146, 2003.
SILVEIRA, L.C.; McMANUS, C.; MASCIOLI, A.S. et al. Fatores ambientais e parâmetros genéticos para características produtivas e reprodutivas em um rebanho Nelore no estado do Mato Grosso do Sul. Rev. Bras. Zootec., v.33, p.1432-1444, 2004.

TOELLE, V.D.; ROBISON, O.W. Estimates of genetic correlations between testicular measurements and female reproductive traits in cattle. J. Anim. Sci., v.60, p.89-100, 1985.

VARGAS, C.A.; ELZO, M.A.; CHASE JR., C.C. et al. Estimation of parameters for scrotal circumference, age at puberty in heifers, and hip height in Brahman cattle. J. Anim. Sci., v.76, p.2536-2541, 1998. 